\title{
Is Allogeneic Blood Transfusion a Risk Factor for Sternal Dehiscence Following Cardiac Surgery? A Prospective Observational Study
}

\author{
Tomáš VyMAZAL, ${ }^{1}$ MD, Michal HoRÁčEK, ${ }^{1} \mathrm{MD}$, Radim DuŘPEKT, ${ }^{2} \mathrm{MD}$, \\ Marie Hladíková, ${ }^{3}$ Mgr, and Karel Cvachovec, ${ }^{1}$ MD
}

\section{SUMMARY}

Sternal dehiscence following cardiac surgery has a multifactorial etiology. Significant risk factors contributing to sternal dehiscence include chronic obstructive pulmonary disease (COPD), obesity, or re-exploration due to bleeding or pericardial tamponade. We have focused on the role of allogeneic blood transfusion as a factor leading to the poor healing of surgical wounds.

A prospective observational study of 1553 elective and emergency cardiac surgery patients was performed between January 2003 and June 2007. All of the patients enrolled in this study underwent median sternotomy. We studied the relationship between sternal dehiscence following cardiac surgery and the total number of packed red blood cells transfused.

The incidence of sternal dehiscence in the study group was 3.4\%. Diabetic patients did not have a higher incidence of wound dehiscence. Although COPD, ${ }^{2)}$ obesity, ${ }^{2)}$ and re-exploration ${ }^{4)}$ contributed to sternal dehiscence, the number of allogeneic blood transfusions during the perioperative period was an important independent risk factor for sternal dehiscence. Patients with sternal dehiscence received an average of 7.6 transfusion units (TU) of allogeneic blood versus $1.6 \mathrm{TU}$ of allogeneic blood in the group without sternal dehiscence $(P<0.00005)$. The dehiscence affected patients without any other significant risk factor who received 6 or more TU, or patients with at least one significant risk factor who received 4 or more TU of allogeneic blood.

According to our results, the total amount of allogeneic blood transfused is an important risk factor contributing to sternal dehiscence. Regardless of other risk preconditions, the transfusion of 6 or more TU could result in sternal dehiscence following cardiac surgery. (Int Heart J 2009; 50: 601-608)

Key words: Sternal dehiscence, Immune modulation, Blood transfusion, Cardiac surgery

SINCE $1956,{ }^{1)}$ median sternotomy has been the most frequently used surgical

From the Departments of ${ }^{1}$ Anesthesiology and Intensive Care Medicine and ${ }^{2}$ Cardiovascular Surgery, University Hospital Motol, Prague, ${ }^{3}$ Medical Informatics, $2^{\text {nd }}$ Faculty of Medicine, Charles University, Prague, Czech Republic.

Address for correspondence: Tomáš Vymazal, MD, Department of Anesthesiology and Intensive Care Medicine, University Hospital Motol, V Úvalu 84, 15006 Prague 5, Czech Republic.

Received for publication September 22, 2008.

Revised and accepted April 27, 2009. 
approach to the heart. Sternotomy wound healing difficulties occur in 1-5\% of cases and are associated with significant patient suffering, prolonged hospital stay, and increased costs. They can be categorized as either superficial lesions involving only soft tissues (skin and subcutaneous) or sternal dehiscences with or without infection.

The most important risk factor for sternal dehiscence is obesity. Other risk factors include diabetes mellitus, COPD, ACE inhibitor use, bilateral mammary artery harvesting, emergency surgery, or mediastinal re-exploration. Their importance is viewed differently and conflicting results are widely discussed. ${ }^{2,4)}$

Cardiac surgery is associated with significant bleeding and blood loss. This lost blood is often replaced by allogeneic blood transfusion. Blood transfusion, however, can lead to adverse effects such as volume overload, febrile reaction, and allergy. It is also associated with many risks, namely hemolytic reaction, infection transmission, or the possibility of immunomodulation. The role of allogeneic blood transfusion as a possible strong immunomodulating agent has been widely discussed recently., ${ }^{5,6,19)}$

Allogeneic blood transfusion represents a possible risk for its recipients. The immunomodulation affects in-hospital morbidity and mortality, ${ }^{7,817,19)}$ increases the risk of infectious complications, ${ }^{9-12,19)}$ prolongs hospitalization, ${ }^{11,13)}$ and increases costs. The restrictive approach to allogeneic blood transfusion should always be adhered to, ${ }^{19)}$ and the guidelines for alternatives to blood transfusion should be followed. ${ }^{14-16,19)}$ The operation should also be avoided in anemic patients. $^{18,20)}$

Our study was designed to evaluate the role of allogeneic blood transfusion in sternal wound dehiscence following median sternotomy in patients who underwent cardiac surgery.

\section{Methods}

Cohort evaluation of all consecutive (ie, elective as well as emergency) patients undergoing cardiac surgery (both off-pump and cardiopulmonary bypass) using median sternotomy at our institution between January 2003 and June 2007 was performed (Table I). Clinical data were collected prospectively and entered into the clinical database. Data analysis was performed retrospectively. The informed consent of patients was not required as follow-up and data analysis is part of the departmental quality management program. This study was approved by the Hospital Institutional Review Board.

Anesthesiological and surgical management including cardiopulmonary bypass were performed in accordance with standard practice in their entirety. Preoperative chest hair removal was performed by manual shaving the day be- 
Table I. Baseline and Clinical Patient Characteristics

\begin{tabular}{lrrr}
\hline & $\begin{array}{c}\text { Sternal dehiscence } \\
(n=53)\end{array}$ & $\begin{array}{c}\text { Without sternal } \\
\text { dehiscence }(n=1500)\end{array}$ & $P$ \\
\hline Age, years & $67 \pm 14$ & $65 \pm 33$ & 0.103 \\
BMI, kg.m ${ }^{-2}$ & $27.6 \pm 3.4$ & $26.5 \pm 3.6$ & 0.031 \\
Diabetes, $n(\%)$ & $28(52.8 \%)$ & $643(42.9 \%)$ & 0.272 \\
COPD, $n(\%)$ & $17(32.3 \%)$ & $184(12.3 \%)$ & 0.033 \\
Left ventricular ejection fraction, $\%$ & 47 & 48 & 0.152 \\
EuroSCORE & $4.8 \pm 1.2$ & $9.7 \pm 1.3$ & 0.098 \\
LIMA + RIMA, $n(\%)$ & $2(4 \%)$ & $790 \pm 560 \mathrm{~mL}$ & 0.324 \\
Blood loss, mL & $1480 \pm 720 \mathrm{~mL}$ & $127(8.5 \%)$ & $<0.0002$ \\
Re-exploration, $n(\%)$ & $9(17.6 \%)$ & 7.5 & $<0.0002$ \\
RBC administered, TU & 7.6 & 0.005 \\
\hline
\end{tabular}

BMI indicates body mass index; COPD, chronic obstructive pulmonary disease; EuroSCORE, European System for Cardiac Operative Risk Evaluation; LIMA, left internal mammary artery; RBC, red blood cell; RIMA, right internal mammary artery; and TU, transfusion unit.

Table II. Symptoms of Sternal Dehiscence

\begin{tabular}{lcc}
\hline Symptom & Number & Percentage \\
\hline Sternal instability & 51 & $96 \%$ \\
Painful deep sternal palpation & 47 & $89 \%$ \\
Positive cultivation & 46 & $88 \%$ \\
Local redness & 41 & $77 \%$ \\
Pus secretion & 11 & $21 \%$ \\
\hline
\end{tabular}

fore the operation in elective cases, or just before the operation in emergencies. Antibiotic prophylaxis was administered at the introduction of anesthesia, ie, 30-60 minutes before the start of operation. Broad spectrum penicillins or 1st or 2nd generation cephalosporins were given for 24 to 48 hours depending on the type of procedure. Perioperative glucose management followed departmental guidelines valid at the time of the study, ie, insulin was given when glycemia exceeded $180 \mathrm{mg} \%$ (10 mmol/L). All transfusion units of red blood cells were administered through a deleucotizing filter. Mammary arteries were harvested exclusively as pedicled grafts. Closure of the sternum was (based on the surgeon's preference) by either 8, 6-gauge stainless-steel wire stitches going through-andthrough the bone, or by figure-of-eight wire stitches going again through-andthrough the bone. The amount of blood lost was assessed regularly every hour and the indication for re-exploration was determined according to Barrat-Boyes criteria or when pericardial tamponade was diagnosed.

The sternal wound was assessed during the patient's stay in hospital and again during a scheduled visit 6 weeks after discharge. Sternal dehiscence was diagnosed by clinical and/or radiological assessment. Clinical signs of sternal dehiscence included local redness or an upswing of temperature, pus secretion, 
Table III. Classification of Mediastinitis in Patients Undergoing Cardiopulmonary Bypass

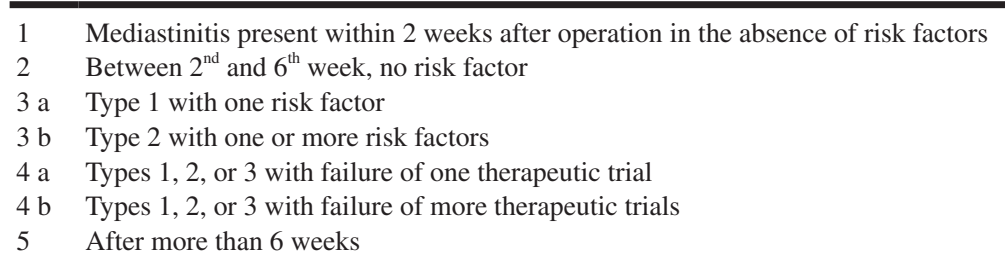

El Oakley RM, Postoperative Mediastinitis: clasification and management, Ann Thorac Surg 1996; 61: 1030-6.

Table IV. Demonstrated Risk Factors and Their Statistical Power

\begin{tabular}{ll}
\hline Risk factor & Significance \\
\hline Diabetes & $P=0.272$ \\
Age & $P=0.103$ \\
COPD & $P=0.033$ \\
BMI & $P=0.031$ \\
Re-exploration & $P<0.002$ \\
Dehiscence developed in patients with no risk factor mentioned & $P<0.00004$ \\
$\quad$ above received 6 $\geq$ TU ABT & $P<0.00006$ \\
Dehiscence developed in patients with risk factors $\geq 1$ mentioned & \\
$\quad$ above received $4 \geq$ TU ABT & $P<0.00005$ \\
\hline Number of ABT TU &
\end{tabular}

tenderness or pain on palpation, and sternal instability (Table II). Infection associated with sternal dehiscence was confirmed by positive microbiological cultivation of mediastinal tissue or fluid. Sternal dehiscence was further classified according to the El Oakley classification (Table III).

The following baseline characteristics were recorded: age, sex, obesity defined by a body mass index (BMI) above 30 (BMI is body weight in kilograms divided by body height in centimeters squared), diabetes (medication with a peroral antidiabetic agent and/or insulin), COPD (regular use of bronchodilators and/or steroids per inhalation or appropriate systemic medication), left ventricular ejection fraction, EuroSCORE, and previous sternotomy. Perioperative parameters included the use of mammary grafts, blood loss, number of red blood cell transfusion units administered during the hospital stay, and re-exploration due to bleeding.

Data are presented as the mean \pm standard deviation or percentage. Both groups, ie, the group with sternal dehiscence versus the group without sternal dehiscence, were compared using the Mann-Whitney test or $\chi^{2}$ test, as appropriate. Statistical analysis was done using SPSS version X (SPSS, Chicago, Ill., USA) and a $P$ value of 0.05 was considered statistically significant. 


\section{Results}

Cohort analysis involved 1553 patients, 1500 without and 53 with sternal dehiscence. The incidence of sternal dehiscence was thus $3.4 \%$. The baseline and perioperative clinical characteristics are summarized in Table I. Patients with sternal dehiscence tended to be older, demonstrated a greater inclination towards obesity (BMI 27.6 versus BMI 26.5; $P=0.031$ ), and were more likely to suffer from COPD $(32.3 \%$ versus $12.3 \%$; $P=0.033)$. The incidence of diabetes was high in both groups, but the difference was not statistically significant (52.8\% versus $42.9 \%$; $P=0.272$ ). Left ventricular ejection fraction as well as the operative risk as assessed by EuroSCORE were identical in both groups. Bilateral mammary artery harvesting was quite rare mainly due to the higher age of CABG patients, who represented roughly two thirds of the study population, and the high proportion of diabetic patients. The amount of blood loss in the group with sternal dehiscence was almost twice as high as in the other group, which is also reflected by the number of administered packed red blood cells. Patients who required re-exploration of the chest due to postoperative bleeding and/or tamponade had sternal dehiscence twice as frequently as patients not requiring re-exploration $(17.6 \%$ versus $8.5 \%$; $P<0.002)$. There was no statistically significant difference in blood loss among different surgeons conducting the procedures.

On average, 7.6 transfusion units (TU) of allogeneic blood were administered to patients with sternal dehiscence versus 1.6 TU of allogeneic blood in patients without sternal dehiscence $(P<0.00005)$. The dehiscence affected patients without any other significant risk factor who received 6 or more TU, or patients with at least one significant risk factor who received 4 or more TU of allogeneic blood (Table IV).

\section{DisCUSSION}

The number of administered allogeneic blood transfusion units as a risk factor in patients who suffer sternal dehiscence has yet to be acknowledged. The immunomodulatory effect of blood transfusion was recently widely discussed $^{5-8,17,19)}$ and also demonstrated in clinical practice in orthopedic surgery by Weber, et al who observed problems with wound healing in $31 \%$ of transfused patients versus $18 \%$ of the nontransfused group $(P<0.05)$ with allogeneic blood transfusion being the only significant predictor for the development of woundhealing disturbances. ${ }^{3)}$ Our study provides evidence that the transfusion of 6 or more allogeneic blood TU could result in sternal dehiscence following cardiac surgery regardless of other risk preconditions. Furthermore, our study confirmed 
that the generally appreciated risk factors of COPD and obesity contribute to sternal wound dehiscence following cardiac surgery in contrast to diabetes mellitus type II (DM II), the impact of which was not statistically significant.

Our study targeted the total number of allogeneic blood transfusion units administered regardless of whether patients were re-explored. ${ }^{4,10,19)}$ All of the surgical procedures were performed by any one of three senior surgeons using standard techniques. None of the patients in the study period suffered from low cardiac output. Nevertheless, the role of a low dose of norepinephrine infusion in a few patients was taken into account. All of the allogeneic blood transfusion units were between 7 and 25 days old. Some authors suggest that the length of storage of blood products could be relevant in the postoperative period. ${ }^{8,9,12,19)}$ Our study did not investigate such data.

There is no doubt about the negative impact of DM II and high blood sugar levels on both macrocirculation and microcirculation. The proportion of diabetics in our study group was very high indeed (see Table I), and the severity of the diabetes varied. Our results show that DM II did not contribute to a greater incidence of sternal dehiscence. ${ }^{2)}$ The reason appears to be effective blood sugar level regulation in patients during hospitalization.

$\mathrm{COPD}^{2)}$ is a very common comorbidity affecting postoperative recovery not only in cardiac surgery patients. The bone and soft tissue sutures are strained by intense coughing and efforts to expectorate viscid mucus during the recovery period, leading to incomplete healing of the sternum. Our results confirm that COPD increases the incidence of sternal dehiscence following cardiac surgery.

Overweight and obese ${ }^{2)}$ people are increasingly more prevalent in the present day population. As fat tissue is poorly vascular, wound healing is compromised. We can confirm that obesity ${ }^{2}$ is a contributing factor to the development of sternal dehiscence following cardiac surgery.

Our study also confirms that re-exploration of the chest ${ }^{4,10,19)}$ due to excessive postoperative bleeding or pericardial tamponade is also a serious and relevant risk factor leading to sternal wound dehiscence following cardiac surgery.

Female gender, age, both LIMA and RIMA harvesting, low ejection fraction of the left ventricle, and high EuroSCORE ${ }^{18)}$ are commonly considered to be risk factors for poor healing of the sternum as well. In our group of patients, there was no significant difference in the incidence of sternal dehiscence as related to ejection fraction of the left ventricle and frequency of both LIMA and RIMA harvesting. The EuroSCORE of patients suffering from sternal dehiscence was slightly higher, appearing to support some authors, ${ }^{14,17,20)}$ but this difference was not statistically significant.

In conclusion, the total number of allogeneic blood transfusion units is significant as one of the independent risk factors contributing to sternal dehiscence. 
Regardless of other risk preconditions, transfusion of 6 or more allogeneic blood TU can result in sternal dehiscence following cardiac surgery.

\section{REFERENCES}

1. Julian OC, Lopez-Belio M, Dye WS, Javid H, Grove WJ. The median sternal incision in intracardiac surgery with extracorporeal circulation; a general evaluation of its use in heart surgery. Surgery 1957; 42: 753-61.

2. Risk factors for deep sternal wound infection after sternotomy: a prospective, multicentre study. J Thorac Cardiovasc Surg 1996; 111: 1200-7.

3. Weber EW, Slappendel R, Prins MH, van der Schaaf DB, Durieux ME, Strümper D. Perioperative blood transfusions and delayed wound healing after hip replacement surgery: effects on duration of hospitalization. Anesth Analg 2005; 100: 1416-21.

4. Karthik S, Grayson AD, McCarron EE, Pullan DM, Desmond MJ. Reexploration for bleeding after coronary artery bypass surgery: risk factors, outcomes, and the effect of time delay. Ann Thorac Surg 2004; 78: 527-34. (Review)

5. Raghavan M, Marik PE. Anemia, allogenic blood transfusion and immunomodulatinon in the critically ill. Chest 2005; 127: 295-307. (Review)

6. Brand A. Immunological aspects of blood transfusions. Transpl Immunol 2002; 10: 183-90. (Review)

7. Koch CG, Li L, Duncan AI, et al. Morbidity and mortality risk associated with red blood cell and blood-component transfusion in isolated coronary artery bypass grafting. Crit Care Med 2006; 34 : 1608-16.

8. Vamvakas EC, Carven JH. Length of storage of transfused red cells and postoperative morbidity in patients undergoing coronary artery bypass graft surgery. Transfusion 2000; 40: 101-9.

9. Rogers MA, Blumberg N, Saint SK, Kim C, Nallamothu BK, Langa KM. Allogeneic blood transfusions explain increased mortality in women after coronary artery bypass graft surgery. Am Heart $\mathrm{J}$ 2006; 152: 1028-34.

10. Gårdlund B, Bitkover CY, Vaage J. Postoperative mediastinitis in cardiac surgery - microbiology and pathogenesis. Eur J Cardiothorac Surg 2002; 21: 825-30.

11. Sirvinskas E, Lenkutis T, Raliene L, Veikutiene A, Vaskelyte J, Marchertiene I. Influence of residual blood autotransfused from cardiopulmonary bypass circuit on clinical outcome after cardiac surgery. Perfusion 2005; 20: 71-5.

12. Vamvakas EC, Carven JH. Transfusion and postoperative pneumonia in coronary artery bypass graft surgery: effect of the length of storage of transfused red cells. Transfusion 1999; 39: 701-10.

13. Vamvakas EC, Carven JH. RBC transfusion and postoperative length of stay in the hospital or the intensive care unit among patients undergoing coronary artery bypass graft surgery: the effects of confounding factors. Transfusion 2000; 40: 832-9.

14. Walsh TS, Saleh EE. Anaemia during critical illness. Br J Anaesth 2006; 97: 278-91. (Review)

15. Leal-Noval R, Muñoz M, Páramo JA, García-Erce JA. Spanish consensus statement on alternatives to allogeneic transfusion: the 'Seville document'. TATM 2006; 8: 178-202.

16. Vymazal T. Can epoietin decrease number of allogeneic blood transfusions in cardiac patients ? VI. Congress of NATA 2005; Poster

17. Ranucci M. Allogeneic blood transfusion and infections after cardiac surgery. Am Heart J 2007; 153: e21.

18. Kulier A, Levin J, Moser R, et al. Impact of preoperative anemia on outcome in patients undergoing coronary artery bypass graft surgery. Circulation 2007; 116: 471-9.

19. Gunst MA, Minei JP. Transfusion of blood products and nosocomial infection in surgical patients. Curr Opin Crit Care 2007; 13: 428-32. (Review)

20. Gardner TJ. To transfuse or not to transfuse. Circulation 2007; 116: 458-60. 